\title{
SINDROME DE BURNOUT Y SATISFACCION LABORAL EN EL PERSONAL SANITARIO DE ESPAÑA Y ARGENTINA
}

\section{BURNOUT SYNDROME AND JOB SATISFACTION IN SPANISH AND ARGENTINEAN HEALTH WORKERS}

Hernández Gijón, M., Jiménez Ortega, A., Llergo Re, M., Mazzoni Catterino, L.A. y Perandrés Gómez, A.M. Universidad de Granada

Artículo dirigido por: Inmaculada Valor Segura. Departamento de Psicología Social. Universidad de Granada

Fecha de recepción: 19 de marzo de 2014.

Fecha de revisión: 19 de marzo de 2014.

Fecha de aceptación: 11 de abril de 2014.

\section{RESUMEN}

Se realizó un estudio transversal sobre Burnout y satisfacción laboral a 107 profesionales del ámbito sanitario hispanohablantes de España y Argentina. Médicos, enfermeros y auxiliares respondieron al cuestionario MBI (Maslach Burnout Inventory) y al Inventario de Satisfacción Laboral (ISL *66). Los objetivos del estudio fueron, por una parte, investigar la prevalencia de Burnout en España y Argentina, así como también, comprobar si existe relación entre el Burnout y la Satisfacción Laboral. Se halló una mayor prevalencia de Burnout en el personal sanitario español frente al argentino, y no se encontró correlación entre Burnout y satisfacción laboral en el personal sanitario de ambos países. Una posible explicación de estos resultados se encuentra en la coyuntura macroeconómica de los países en los últimos años.

Palabras clave: Burnout, Satisfacción laboral, Sanitario.

\begin{abstract}
A cross-sectional study of burnout and job satisfaction for 107 health professionals of the speaking area of Spain and Argentina was conducted. Doctors, nurses and auxiliary answered two questionnaires: the MBI (Maslach Burnout Inventory) and job satisfaction inventory (ISL * 66). The aims of the study were to investigate the prevalence of Burnout in Spain and Argentina and to study if there is a relation between Burnout and Job Satisfaction. It was found more prevalence of Burnout in Spanish health professionals than in Argentinean and it was not found any correlation between Burnout and Job Satisfaction in both countries. A possible explanation of these results could be found in the macroeconomic conjuncture of both countries.
\end{abstract}

Key Words: Burnout, Job satisfaction, Health professional.

\section{INTRODUCCIÓN}

El término Burnout acuñado por Fredeunberger en 1.974, adquiere relevancia con los estudios llevados a cabo por Maslach y Jacksons, quienes en su modelo multidimensional definieron dicho síndrome como una respuesta inadecuada al estrés emocional crónico, constituido por tres dimensiones; el cansancio emocional, (CE); la despersonalización (DP) y la incompetencia personal (FRP).

En lo que a su clasificación se refiere, el Burnout, entendido como fatiga, se ubica en la categoría de "fatiga emocional", causada por un agravado estrés y caracterizada por una disminución o embotamiento de las respuestas emocionales (Ramos, Peiró y Ripoll, 1996). Schwartzmann (2004), por su parte, define el Burnout como una 
"enfermedad profesional". En el contexto de las organizaciones sanitarias son estresores especialmente relevantes para el desarrollo del síndrome, las relaciones sociales de los profesionales de la salud con los pacientes y sus familiares. Algunos autores determinan que se trata de un tipo particular de mecanismo de afrontamiento y autoprotección frente al estrés generado por la relación profesional-cliente, así como por la relación profesional-organización. De esta forma, señalan que dicha respuesta aparece cuando fallan las estrategias funcionales de afrontamiento que suelen emplear los profesionales de la salud, lo que supone una sensación de fracaso profesional y en las relaciones interpersonales con los pacientes (Gil-Monte y Peiró, 1997; Gil-Monte, Peiró y Valcárcel, 1998).

Según la O.M.S., los trabajadores son el recurso más importante que posee un sistema de salud. Dentro de los riesgos laborales de carácter psicosocial, el Burnout ocupa un lugar importante, pues constituye una de las principales causas de deterioro de las condiciones de trabajo, así como una fuente de accidentabilidad y absentismo. Diversos autores documentan que este síndrome afecta más a profesiones que requieren un contacto directo con personas, entrega e implicación (enfermeros, médicos, trabajadores sociales, profesores, policías). Por consiguiente, la categoría profesional se relaciona con la incidencia del síndrome, detectándose una serie de áreas de trabajo con mayor prevalecía que son, los servicios de urgencia, la Unidad de Cuidados Intensivos (UCl) y el área de oncología. Prosiguiendo con los aspectos epidemiológicos descritos en la literatura, parece no existir acuerdo unánime entre los distintos autores, si bien existe cierta concomitancia para algunas variables. La edad no parece influir. Según la variable sexo, serían las mujeres sanitarias las más afectadas, se sospecha que por la doble carga de trabajo familiar y profesional.

Otros factores más discutidos son la antigüedad profesional, el salario, estado civil, la sobrecarga laboral, la existencia o no de hijos, los cambios de turnos y los horarios laborales.

Álvarez y Fernández (1991) reconocen asociados al Burnout síntomas psicosomáticos, como fatiga crónica, cefaleas, úlcera gástrica y pérdida de peso. También se manifiestan alteraciones conductuales, como ausentismo laboral, consumo de alcohol y drogas, comportamientos de alto riesgo y conflictos. Además de esto, se denota la aparición de alteraciones emocionales tales como distanciamiento social, irritabilidad, depresión, dificultades de concentración, e incluso ideación suicida. Para terminar, señalan que se pueden presentar negación de las emociones y otros mecanismos de defensa.

Asimismo, no hemos de olvidar que el síndrome de Burnout provoca insatisfacción en el área laboral. Se considera la satisfacción laboral como una actitud, pues hace alusión a conductas y opiniones del operario hacia sus tareas. Se ha definido, por tanto, como "actitudes laborales positivas", las cuales sugieren "sentimientos de satisfacción cuando se piensa o habla del propio puesto de trabajo, aunque diversos aspectos del trabajo pueden resultar en tipos diferentes de sentimientos" (Hulin, 1991). Existen dos formas de acercarse al estudio de la satisfacción laboral, aquella en la que se prioriza el aspecto afectivo de la actitud (Bravo, Peiró y Rodríguez, 1996; Fiske y Taylor, 1991,), y la que traspasa la barrera de los sentimientos, considerándola como una actitud generalizada, de tal forma que se contemplan los tres factores que constituyen una actitud (Bravo et al., 1996). Es importante resaltar que, en atención sanitaria, la satisfacción laboral se iguala en importancia a la preparación científicotécnica del profesional o a la disposición de una determinada tecnología. El grado de calidad y de eficacia de los servicios prestados en un sistema sanitario está en relación directa con el nivel de satisfacción de los profesionales que en él trabajan. Po todo ello, la satisfacción laboral puede estar relacionada con los niveles del síndrome de Burnout (Cherniss, 1980, Burke y Greenglass, 1989; Jiménez, 2004). Otros 
estudios también plantean una relación estrecha entre satisfacción laboral y Burnout (Jiménez, 2004; Gil, Alcover, Peiró, 2005; Grau et al., 2005; Molina, García y Alonso, 2003); en todos ellos se demuestra un alto nivel de significación estadística en la correlación negativa entre ambos factores, es decir, a mayor puntaje de los componentes del síndrome de Burnout, menor es la satisfacción laboral.

De igual forma, algunos investigadores consideran los aspectos sociales, económicos y culturales relevantes en lo que a la génesis y repercusiones del síndrome respecta, por lo se recomienda el estudio del Burnout en diferentes poblaciones. Así, se ha podido observar que la sociedad occidental, generadora de competitividad y materialismo, se ve influenciada por factores que la predisponen a experimentar Burnout (Mingote Adam, 1977). Otros consideran que su afección no sólo repercute a sociedades del bienestar y que se trata de un problema transnacional y transcultural (Gil-Monte, 2008). Grau et al., (2009) encontraron un claro predominio del síndrome de "quemarse por el trabajo" en España y Argentina frente a otros países de habla hispana.

Con todo lo dicho, teniendo en cuenta la bibliografía previa y el interés que suscita el estudio transnacional, proponemos como objetivo principal de nuestra investigación estudiar la prevalencia del síndrome de Burnout en España y Argentina, así como su relación con la insatisfacción laboral, la cual parece ser provocada, entre otros factores, por dicho síndrome. Las hipótesis en cuestión serán, primeramente, una mayor prevalencia del síndrome en España respecto a Argentina; por otra parte, la segunda hipótesis será que cuanto más nivel de Burnout se presente, mayor será la insatisfacción laboral.

\section{MÉTODO}

\section{Diseño}

Burnout, en sus 3 dimensiones (CE, DP Y RP), Nivel de Satisfacción Laboral en sus cuatro dimensiones (EP, DCA, COM, SAL) y País de Nacimiento (España/Argentina).

\section{Participantes}

La muestra de estudio estaba compuesta de 107 participantes profesionales del sector sanitario hispanohablantes, residentes en España y Argentina, en una proporción de 68 españoles y 39 argentinos. El muestreo fue por conveniencia.

La muestra perteneciente a España se obtuvo de los siguientes centros: centro de salud de la Chana, centro de urgencias de Motril, hospital Ruíz de Alda, hospital Virgen de las Nieves, hospital San Cecilio, todos ellos de la provincia de Granada y hospital Reina Sofía de Córdoba Capital.

Mientras que la muestra argentina se obtuvo de estos otros centros: Sanatorio del Norte SRL, Hospital Escuela General San Martín, Hospital J.R Vidal, Hospital Pediátrico Juan Pablo II, todos ellos de Corrientes Capital.

\section{Material}

MBI (Maslach Burnout Inventory)

Para medir el grado de Burnout en los participantes se utilizó el MBI (Maslach y Jackson, 1986). Este instrumento consta de 22 ítems con 7 opciones de respuesta según la frecuencia con la que se experimentan ciertas sensaciones en el trabajo, puntuando de 0 a 6 para cada uno de los ítems. El cuestionario tiene en cuenta tres dimensiones de dicho constructo: Cansancio Emocional (CE), evaluado con 9 ítems, que considera la disminución de la capacidad emocional a causa de estar agotado en el trabajo; Despersonalización (DP), evaluada mediante 5 ítems, describe una 
deshumanización en el trato a los pacientes; y Realización Personal (RP) evaluada con 8 ítems, que se relaciona con el éxito laboral autopercibido y, en contraposición con los dos anteriores, valores bajos en este índice son indicativos de padecimiento de Burnout.

\section{INVENTARIO DE SATISFACCIÓN LABORAL (ISL *66).}

Para medir la satisfacción laboral se hizo uso del ISL (Galicia y Hernández, 2000). Este inventario consta de 66 ítems con 5 opciones de respuesta según el grado de acuerdo con la situación personal laboral de cada participante. Explora cómo se siente cada participante en relación con su trabajo, valorando las condiciones laborales de relación con compañeros y jefes, el cumplimiento de objetivos personales, la estabilidad y el agotamiento laboral.

Ambos instrumentos se presentaron en versión online, y se cumplimentaron en ordenadores facilitados por los experimentadores.

\section{RESULTADOS}

Se analizaron los resultados con el programa estadístico SPSS. En la tabla 1 se han descrito los estadísticos de Burnout y Satisfacción Laboral de la muestra dividida por países.

Tabla 1. Media (M) y Desviación Típica (DT) de las variables de Burnout y Satisfacción Laboral ( $n=68$ España y $n=39$ Argentina).

\begin{tabular}{lll}
\hline Cansancio emocional & $M$ & $D T$ \\
\hline España & 31,68 & 7,41 \\
Argentina & 7,00 & 3,62 \\
\hline Despersonalización & $M$ & $D T$ \\
\hline España & 14,85 & 4,56 \\
Argentina &, 53 &, 97 \\
\hline Realización personal & $M$ & $D T$ \\
\hline España & 19,86 & 7,38 \\
Argentina & 45,87 & 2,55 \\
\hline Evaluación institucional del personal & $M$ & $D T$ \\
\hline España & 53,31 & 11,74 \\
Argentina & 71,54 & 4,36 \\
\hline Desarrollo, capacitación y actualización del personal & $M$ & $D T$ \\
\hline España & 84,69 & 14,58 \\
Argentina & 108,62 & 9,93 \\
\hline Comunicación & $M$ & $D T$ \\
\hline España & 60,00 & 13,10 \\
Argentina & 81,08 & 5,24 \\
\hline Salud y condiciones laborales & $M$ & $D T$ \\
\hline España & 11,62 & 3,14 \\
Argentina & 12,51 & 2,41
\end{tabular}


En la tabla 2 se analizó la Satisfacción laboral; examinada con sus respectivas dimensiones (EP, DCA, COM, SAL) en correlación con las dimensiones del Burnout (CE, DP, RP) y dividido por países. Sólo se encontró correlación significativa en la dimensión de Salud y Condiciones Laborales (SAL) con Realización personal (RP) en el personal Español. En el resto de dimensiones, no se encontraron correlaciones significativas en ninguno de los dos países.

Tabla 2. Correlación de Satisfacción Laboral y Burnout (España y Argentina). Correspondiente a Hipótesis 2.

\begin{tabular}{|c|c|c|c|c|c|c|c|c|c|c|c|c|c|c|}
\hline & $\overline{C E}$ & & $\overline{D P}$ & & $\overline{R P}$ & & EP & & $\overline{D C A}$ & & COM & & $\overline{S A L}$ & \\
\hline & E & A & $E$ & A & E & A & $E$ & A & $\mathrm{E}$ & A & E & A & E & A \\
\hline$\overline{C E}$ & - & - & & & & & & & & & & & & \\
\hline DP &, $58\left(^{\star \star *}\right)$ &, $50(* *)$ & - & - & & & & & & & & & & \\
\hline RP &,$- 313\left({ }^{\star *}\right)$ &,$- 514(* *)$ &,$- 249\left(^{*}\right)$ & 0,02 & - & - & & & & & & & & \\
\hline EP & $-0,04$ & 0,02 & 0,14 & 0,01 & $-0,13$ & $-0,08$ & - & - & & & & & & \\
\hline DCA & $-0,11$ & 0,02 & 0,05 & 0,10 & $-0,08$ & 0,16 &, $844\left({ }^{* *}\right)$ & $8499^{(* *}$ & - & - & & & & \\
\hline com & $-0,05$ & $-0,06$ & 0,11 & 0,05 & $-0,17$ & 0,14 &, $850\left({ }^{* *}\right)$ &, $833\left(^{(* *}\right)$ &, $906\left(^{\star \star}\right)$ &, $\left.8511^{\star \star *}\right)$ & - & - & & \\
\hline SAL & 0,04 & 0,00 & $-0,12$ & 0,26 &, $3311^{(* *}$ & 0,30 &,$- 49\left(^{\star *}\right)$ & 0,22 &,$- 50\left(^{\star *}\right)$ & , $4400^{(* *}$ &,$- 54\left(^{(*)}\right)$ & 0,29 & & \\
\hline
\end{tabular}

CE: Cansancio Emocional, DP: Despersonalización, RP: Realización Personal. EP: Evaluación del personal, DCA: Desarrollo, Capacitación y actualización del personal. C: comunicación. SAL: Salud y condiciones laborales.

E: España, A: Argentina. 


\section{DISCUSIÓN}

A través de los datos observados comprobamos que se cumple la primera hipótesis del estudio, encontrándose un mayor nivel de Burnout en el personal sanitario español frente al argentino. Respecto a las dimensiones correspondientes al síndrome, se encontró una puntuación significativamente más alta en la población sanitaria española de las dimensiones de Cansancio Emocional y Despersonalización, en comparación con la argentina. No obstante, en la dimensión de Realización Personal, hallamos una puntuación significativamente más alta en la población sanitaria argentina.

En lo que a la segunda hipótesis se refiere, no se encontraron correlaciones significativas entre las dimensiones correspondientes al Burnout y las de Satisfacción Laboral en ninguno de los dos países, exceptuando, eso sí, la dimensión de Realización Personal con la de Salud y Condiciones laborales, la cual resultó significativa en la población sanitaria española.

Una posible explicación de las discrepancias en el nivel de Burnout encontradas en ambos países residiría en la realidad socio-económica de cada uno. En países con un grado de bienestar inferior, las condiciones laborales/personales más duras y difíciles, son apreciadas como menos estresantes (Argentina). Quizás esto sea debido a que los individuos de estas poblaciones suelen tener menos expectativas y ser más conformistas, atribuyendo el agotamiento laboral al contexto macroeconómico, más que a las condiciones laborales, al contrario que en aquellos donde existe un mayor bienestar y concienciación de los derechos de los trabajadores (España). Dado que nuestro estudio, por su diseño, no consideró las características de las diferencias encontradas, serían necesarias futuras investigaciones a nivel social y antropológico para su constatación.

En un metanálisis realizado por Atance (1997), en el que fueron comparados veinte estudios en España sobre personal sanitario y Burnout se observa que las medias de nuestro estudio no están dentro de las oscilaciones publicadas (de 18,6 a 29 para CE, de 6,4 a 10,8 para DP y de 28 a 40,6 para RP); siendo en nuestro estudio: CE: 31, 6; DP: 14,85; RP19, 86. Estas diferencias se encuentran en la línea de la explicación anterior: la crisis actual de España, la cual se ha agravado en los últimos cinco años, ha provocado un empeoramiento de las condiciones laborales, lo que ha repercutido en un aumento de Burnout por parte del personal sanitario español.

Una limitación de nuestro estudio corresponde a que actualmente no se dispone de un criterio consensuado para considerar que un caso se corresponde al padecimiento de Burnout. El recoger y analizar los datos con la misma metodología, permitiría realizar comparaciones con otros grupos sanitarios de otros estudios con mayor fiabilidad que realizarlo con diferentes diseños. Así mismo, es importante destacar la posible existencia de un sesgo de selección, ya que parte del estudio dependía del interés del participante.

Con todo lo dicho y, siguiendo la perspectiva de Maslach y Leither (1997), nos parece interesante introducir una nueva perspectiva en estudios futuros conocida como "engagement". Parece constituir un constructo opuesto al Burnout, y saca a relucir algunas cuestiones hasta ahora desconocidas de dicho síndrome. De esta forma, ya que es bien conocido el Burnout, sería relevante, además, explorar aspectos positivos que resulten útiles a la hora de contrarrestar su incidencia, fomentando no sólo aspectos negativos y nocivos del empleo, sino también recursos positivos del mismo con los que se pueda trabajar directa e indirectamente. 


\section{BIBLIOGRAFÍA}

Albaladejo, R., Villanueva, R., Ortega, P., Astasio, P., Calle, M.E. y Domínguez, V. (2004). Síndrome de Burnout en personal de enfermería de un hospital de Madrid. Revista Española de Salud Pública, 78 (4), 505-516.

American Psychiatric Association. (1996). DSM IV. Manual diagnóstico y estadístico de los trastornos mentales. Madrid: Masson.

Atance, J.C. (1997). Aspectos epidemiológicos del Síndrome de Burnout en personal sanitario. Revista Española de Salud Pública, 71 (3), 293-303.

Burke, R.J. \& Greenglass, E.R. (1989). Psychological burnout among men and women in teaching. An examination of the Cherniss model. Human Relations, 42, 261273.

Bustinza, A., López-Herce, J., Carrillo, A., Vigil, M.D., De Lucas, N. y Panadero, E. (2000). Situación de Burnout de los pediatras intensivistas españoles. Anales Españoles de Pediatría, 52 (5), 418-423.

Cherniss, C. (1980). Professional burnout in human service organizations. Nueva York: Praeger Publisher.

Flórez-Lozano, J.A. (1994) Síndrome de estar "quemado". Barcelona: Edikamed.

Freudenberg, H.J. (1974). Staff Burn-out. Journal Social of Issues, 30, 159-165.

Gil, F., Alcover, J.M. y Peiró, J.M. (2005). Work team effectiveness in organizational contexts: Recent research and applications in Spain and Portugal. Bradford, RU. Journal of Managerial Psychology, 20 (4), 193-218.

Gil-Monte, P.R. (2008). El síndrome de quemarse por el trabajo (burnout) como fenómeno transcultural. Información Psicológica, 91-92, 4-11.

Gil-Monte, P.R. y Peiró, J.M. (1997). Desgaste psíquico en el trabajo: el síndrome de quemarse. Madrid: Síntesis.

Gil-Monte, P.R., Peiró, J.M. \& Valcárcel, P. (1998). A model of burnout process development: An alternative from appraisal models of stress. Comportamento Organizacional e Gestao, 4 (1), 165-179.

González, P., Peiró, J.M. y Bravo, M.J. (1996). Calidad de vida laboral. En J.M. Peiró y F. Prieto (Eds.), Tratado de Psicología del trabajo. Vol. II. Aspectos psicosociales del trabajo (pp. 161-186). Madrid: Síntesis.

Grau, A., Flichtentrei, D., Suñer, R., Prats, M. y Braga, F. (2009). Influencia de factores personales, profesionales y transnacionales en el Síndrome de Burnout en personal sanitario hispanoamericano y español (2007). Revista Española de Salud Pública, 83 (2), 215-230.

Grau, A., Suñer, R., García, M.M. (2005). Desgaste profesional en el personal sanitario y su relación con los factores personales y ambientales. Gaceta Sanitaria, 19 (6), 463-470. 
House J.S. (1981). Work stress and social support. Reading, MA: Addison-Wesley.

Hulin, C.L. (1991). Adaptation, persistence, and commitment in organizations. En M.D. Dunnette \& I.M. Hough (Eds.), Handbook of industrial and organizational psychology (pp. 445-506). Palo Alto, CA: Consulting Psychologists Press.

Jiménez, J. P. (2004). Bienestar y satisfacción de los médicos con el ejercicio de su profesión en un sistema de salud en proceso de reforma. Revista médica de Chile, 132 (5), 635-636.

Lomeña, J.A., Campaña, J.M., Nuevo, G., Rosas, D., Berrocal, A. y García, F. (2004). Burnout y satisfacción laboral en Atención Primaria. Medicina de Familia (And), $5(3), 147-155$.

Maslach, C. \& Jackson, S. (1986). Maslach Burnout inventory (2a ed.). Palo Alto, CA: Consulting Psychologist Press.

Mingote, J.C. (1997). Síndrome «burnout», síndrome de desgaste profesional. Monografías de Psiquiatría, 5, 1-44.

Molina, A., García, M.A. y Alonso M. (2003). En relación con el burnout. Medifam, 13, (4), 89-90.

Moreno, B., Bustos, R., Matallana, A. y Miralles, T. (1997). La evaluación del Burnout. Problemas y alternativas. El CBB como evaluación de los elementos del proceso. Revista de Psicología del Trabajo y las Organizaciones, 13 (2), 185207.

Moreno, B., Oliver, C., Pastor, J.C. y Aragoneses, A. (1990). El Burnout, una forma específica de estrés laboral. En V.E. Carballo y G. Buela-Casal (Eds.), Manual de psicología clínica. Madrid: Siglo XXI.

Organización Mundial de la Salud. (1992). CIE-10. Trastornos mentales y del comportamiento. Descripciones clínicas y pautas para el diagnóstico. Madrid: Mediator.

Organización Mundial de la Salud (2000). The World Health Report 2000. Health systems: Improving performance. Extraído el 1 de enero de 2014, de http://www.who.int/whr/2000/en/.

Ramos, J., Peiró, J.M. y Ripoll, P. (1996). Condiciones de trabajo y clima laboral. En J.M. Peiró, y F. Prieto (Eds.), Tratado de Psicología del trabajo. Vol. I: La actividad laboral en su contexto. Madrid: Síntesis.

Rodríguez, A. y Dei, H.D. (2002). Psicosociología de las organizaciones. Buenos Aires: Docencia.

Román, J. (2003). Estrés y Burnout en profesionales de la salud de los niveles primario y secundario de atención. Revista Cubana de Salud Pública, 29 (2), 103-10.

Salanova, M., Schaufeli, W.B., Llorens, S., Peiro, J.M. y Grau, R. (2000). Desde el "burnout" al "engagement": ¿una nueva perspectiva? Revista del Trabajo y de las Organizaciones, $16(2), 117-134$. 
Thomaé, M.N., Ayala, E.A., Sphan, M.S., y Stortti, M.A. (2006). Etiología y prevención del síndrome de burnout en los trabajadores de la salud. Revista de Posgrado de la Vla Cátedra de Medicina, 153, 18-21. 